\title{
Leptonic radiative corrections to elastic deuteron-electron scattering
}

\author{
G. I. Gakh, M. I. Konchatnij, and N. P. Merenkov \\ NSC "Kharkov Institute of Physics and Technology", Akademicheskaya, 1, 61108 Kharkov, Ukraine \\ and V.N. Karazin Kharkiv National University, 61022 Kharkov, Ukraine \\ Egle Tomasi-Gustafsson \\ IRFU, CEA, Université Paris-Saclay, 91191 Gif-sur-Yvette Cedex, France
}

(Received 2 April 2018; revised manuscript received 3 September 2018; published 26 October 2018)

\begin{abstract}
QED first-order radiative corrections to the differential cross section for elastic scattering of deuterons on electrons at rest are calculated. Radiative corrections due to soft- and hard-photon bremsstrahlung from the electron lines, the electron vertex correction, and the electron vacuum polarization are considered. The model-dependent contributions due to the bremsstrahlung from the deuteron and the deuteron vertex correction, depending on its internal structure, are not included. We consider an experimental setup where the final particles are recorded in coincidence and their energies are determined within some uncertainties. Formulas for the relevant kinematical variables, the cross section, and the radiative corrections are derived and numerical results are presented.
\end{abstract}

DOI: 10.1103/PhysRevC.98.045212

\section{INTRODUCTION}

Polarized and unpolarized scattering of electrons off protons and light nuclei has been widely studied since these experiments give information on the internal structure of these particles (for recent reviews, see Refs. [1,2] and references therein).

The recent determination of the proton electromagnetic form factors, using the polarization method [3], shows that for transferred momenta $Q^{2}=-q^{2} \geqslant 1 \mathrm{GeV}^{2}$ the polarized and unpolarized experiments result in inconsistent values of the form factor ratio; see Ref. [4]. This puzzle has given rise to many speculations and different interpretations, as, for example, the two-photon exchange contribution [5-9], suggesting further experiments. Recent experiments searched for evidence of two-photon exchange; see Refs. [10-12]. It is expected that such contribution becomes more important with heavier targets and at small angles $[13,14]$.

In the region of small $Q^{2}$, one can determine the charge radius of the proton and of the light nuclei $\left(r_{c}\right)$, which is one of the fundamental quantities in physics. In this case, the statistical precision on the elastic cross section is not the limiting factor, but systematic effects related to the extrapolation to $Q^{2} \rightarrow 0$ of the cross-section derivative prevent higher precision.

Published by the American Physical Society under the terms of the Creative Commons Attribution 4.0 International license. Further distribution of this work must maintain attribution to the author(s) and the published article's title, journal citation, and DOI. Funded by $S C O A P^{3}$.
Recently, the determination of the proton $r_{c}$ with muonic atoms led to the so-called proton radius puzzle. Experiments on muonic hydrogen by laser spectroscopy lead to the value $r_{c}=0.84087(39)$ [15,16], which is one order of magnitude more precise but smaller by seven standard deviations compared to the average value recommended by the 2010 CODATA review [17].

Different sources of possible systematic errors of the muonic experiments have been discussed. However, no definite explanation of this difference has been given yet (see Refs. [18-20] and references therein).

The deuteron form factors have been also extensively investigated during recent years; see the reviews [21-23]. The precise knowledge of the deuteron charge radius can give additional information about the deuteron internal structure. The authors of Ref. [24] check the contribution from the different coordinate intervals of the deuteron wave function to the radius and found that it was sizable in the large $r$ region. So, they concluded that extrapolation of the wave function at large distances is of great interest. A new method which allows us to fix the percentage of the elusive $D$-state probability, $P_{D}$, from experiments is suggested in Ref. [25]. It uses the dependence of the deuteron charge radius, $r_{d}$, on the deuteron wave function. Therefore, the precise knowledge of $r_{d}$ allows us to determine $P_{D}$ more accurately.

The CREMA Collaboration has just published a value of the radius $r_{d}$ from laser spectroscopy of the muonic deuterium $(\mu d)$ [26],

$$
r_{d}^{\mu d}=2.1256(8) \mathrm{fm},
$$

again more than $7 \sigma$ smaller than the CODATA-2010 value of $r_{d}[27]$

$$
r_{d}^{\text {CODATA-2010 }}=2.1424(21) \mathrm{fm} .
$$


As was noted in Ref. [28], the comparison of the new $r_{d}^{\mu d}$ value with the CODATA-2010 value may be considered inadequate or redundant, because the CODATA values of $r_{d}$ and $r_{p}$ are highly correlated. A pedagogical description of the method to extract the charge radius and the Rydberg constant from laser spectroscopy in regular hydrogen and deuterium atoms is given in Ref. [28]. The principle of determining the deuteron radius from deuterium spectroscopy is analogous to the one described for hydrogen above. However, not all measurements were done for deuterium [28].

In order to reach the smallest values of the transferred momentum to constrain the extrapolation to $Q^{2} \rightarrow 0$, the use of deuteron elastic scattering on atomic electrons (in inverse kinematics) was suggested [29]. Inverse kinematics was proposed in a number of theoretical papers [30-32].

To our knowledge, no experiment was performed yet on proton and deuteron scattering on atomic electrons. Such experiment is in principle possible at the Nuclotron accelerator, in Dubna, where polarized deuteron beams up to 13 $\mathrm{GeV}$ energy can be accelerated [33]. Similar experiments were performed with kaon beams at CERN SPS [34] and with pion beams at Serpukov [35] to determine their radii. Inverse kinematics was also proposed to measure neutron capture cross section of unstable isotopes [36]. For proton and $\alpha$-induced reactions, employing a radioactive ion beam hitting a proton or helium target at rest was suggested. The experiment [37], proposed at CERN, will measure the running of the fine-structure constant in the spacelike region by scattering high-energy muons (with energy $150 \mathrm{GeV}$ ) on atomic electrons, $\mu e \rightarrow \mu e$. The proposed technique is similar to the one described in Refs. [38,39].

In this paper, we extend to $d e$ scattering the approach of Ref. [32] dedicated to proton scattering on atomic electrons. We consider an experimental setup where the scattered deuteron and electron are recorded in coincidence and their energies are determined within uncertainties. We calculate the QED radiative corrections to the leptonic part of interaction. These radiative corrections arise from the soft- and hard-photon bremsstrahlung by the electrons, the electron vertex correction, and the electron vacuum polarization. The contributions due to the bremsstrahlung from the deuteron and the deuteron vertex correction as well the two-photon exchange graph, depending on its internal structure, are not considered. Concerning the hard photon calculation, we followed Ref. [40] for the coordinate system and the angular integration.

\section{FORMALISM}

Let us consider the reaction

$$
d\left(p_{1}\right)+e^{-}\left(k_{1}\right) \rightarrow d\left(p_{2}\right)+e^{-}\left(k_{2}\right),
$$

where the momenta of the particles are indicated in parentheses, and $q=k_{1}-k_{2}=p_{2}-p_{1}$ is the four-momentum of the virtual photon. The reference system is the laboratory (lab) system, where the electron target is at rest.

A general characteristic of all reactions of elastic and inelastic hadron scattering by atomic electrons (that can be considered at rest) is the small value of the momentum transfer square, even for relatively large energies of the colliding particles. The electron mass cannot be neglected in the kinematics and dynamics of the reaction, even when the beam energy is of the order of $\mathrm{GeV}$. The details of the inverse kinematics are given in Ref. [32]. We recall here an useful quantity, the maximum value of the recoil electron energy $\epsilon_{2}$ :

$$
\epsilon_{2 \max }=m \frac{2 E(E+m)+m^{2}-M^{2}}{M^{2}+2 m E+m^{2}},
$$

where $m(M)$ is the electron (deuteron) mass and $E$ is the deuteron beam energy.

In the one-photon exchange (Born) approximation, the matrix element $\mathcal{M}^{(B)}$ of the reaction (1) can be written as

$$
\mathcal{M}^{(B)}=\frac{e^{2}}{q^{2}} j_{\mu} J_{\mu},
$$

where $j_{\mu}\left(J_{\mu}\right)$ is the leptonic (hadronic) electromagnetic current. The leptonic current is

$$
j_{\mu}=\bar{u}\left(k_{2}\right) \gamma_{\mu} u\left(k_{1}\right),
$$

where $u\left(k_{1,2}\right)$ is the spinor of the incoming (outgoing) electron. Following the requirements of Lorentz invariance, current conservation, parity, and time-reversal invariance of the hadronic electromagnetic interaction, the general form of the electromagnetic current for the deuteron (that is a spin-1 particle) is fully described by three form factors. The hadronic electromagnetic current can be written as [41]

$$
\begin{aligned}
J_{\mu}= & \left(p_{1}+p_{2}\right)_{\mu}\left[-G_{1}\left(q^{2}\right) U_{1} \cdot U_{2}^{*}+\frac{1}{M^{2}} G_{3}\left(q^{2}\right)\right. \\
& \left.\times\left(U_{1} \cdot q U_{2}^{*} \cdot q-\frac{q^{2}}{2} U_{1} \cdot U_{2}^{*}\right)\right] \\
& +G_{2}\left(q^{2}\right)\left(U_{1 \mu} U_{2}^{*} \cdot q-U_{2 \mu}^{*} U_{1} \cdot q\right),
\end{aligned}
$$

where $U_{1 \mu}$ and $U_{2 \mu}$ are the polarization four vectors for the initial and final deuteron states. The functions $G_{i}\left(q^{2}\right)$, $i=1,2,3$, are the deuteron electromagnetic form factors, depending only on the virtual photon four-momentum squared $q^{2}=-Q^{2}=\left(k_{1}-k_{2}\right)^{2}=2 m\left(m-\varepsilon_{2}\right)$. Due to the current Hermiticity, these form factors are the real functions in the region of the spacelike momentum transfer.

These form factors are related to the standard deuteron form factors: $G_{C}$ (charge monopole), $G_{M}$ (magnetic dipole), and $G_{Q}$ (charge quadrupole) by the following relations:

$$
\begin{aligned}
G_{M}\left(q^{2}\right) & =-G_{2}\left(q^{2}\right), \\
G_{Q}\left(q^{2}\right) & =G_{1}\left(q^{2}\right)+G_{2}\left(q^{2}\right)+2 G_{3}\left(q^{2}\right), \\
G_{C}\left(q^{2}\right) & =\frac{2}{3} \tau\left[G_{2}\left(q^{2}\right)-G_{3}\left(q^{2}\right)\right]+\left(1+\frac{2}{3} \tau\right) G_{1}\left(q^{2}\right), \\
\tau & =-\frac{q^{2}}{4 M^{2}} .
\end{aligned}
$$

The standard form factors have the following normalization:

$$
G_{C}(0)=1, \quad G_{M}(0)=\frac{M}{m_{N}} \mu_{d}, \quad G_{Q}(0)=M^{2} \mathcal{Q}_{d},
$$



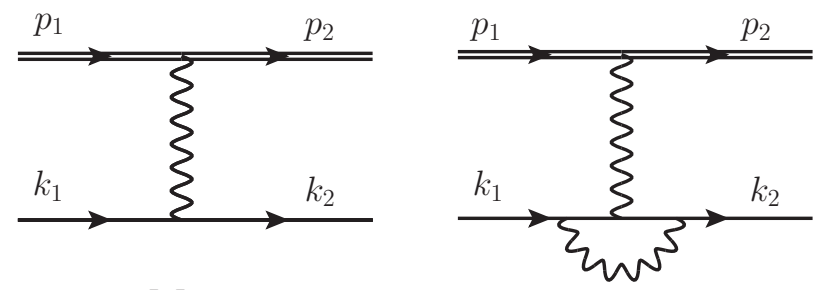

$\mathrm{M}_{0}$

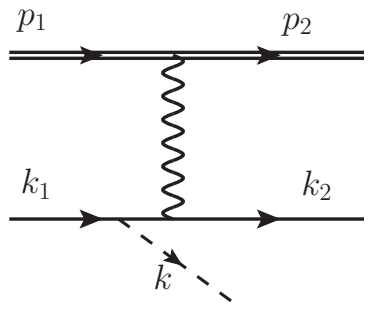

$\mathrm{M}_{\mathrm{s}}$
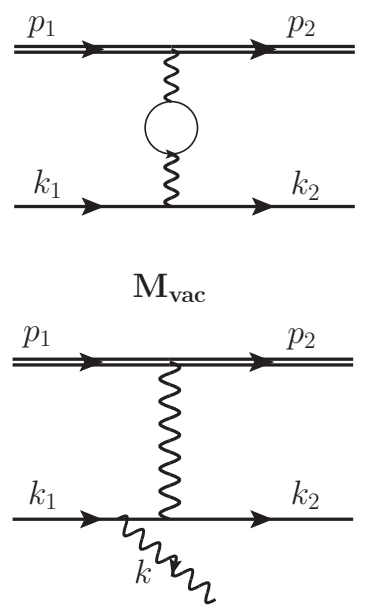

$\mathrm{M}_{\mathrm{h}}$

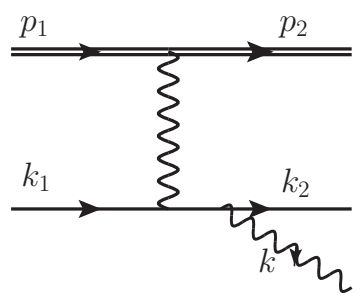

FIG. 1. Feynman's diagrams corresponding to the Born approximation, first-order vertex and photon self-energy corrections (top), initial and final real photon bremsstrahlung, $M_{s}$ (soft) and $M_{h}$ (hard), from the lepton vertex (bottom).

where $m_{N}$ is the nucleon mass, $\mu_{d} / \mu_{N}=0.857$ is the deuteron magnetic moment in the units $1 / 2 m_{N}$, and $\mathcal{Q}_{d}=$ $0.2857 \mathrm{fm}^{2}$ is the deuteron quadrupole moment.

The matrix element squared is written as

$$
\left|\mathcal{M}^{(B)}\right|^{2}=16 \pi^{2} \frac{\alpha^{2}}{q^{4}} L_{\mu \nu} W_{\mu \nu},
$$

where $\alpha=e^{2} /(4 \pi)=1 / 137$ is the electromagnetic fine structure constant. The leptonic $L_{\mu \nu}$ and hadronic $H_{\mu \nu}$ tensors are defined as

$$
L_{\mu \nu}=j_{\mu} j_{\nu}^{*}, \quad H_{\mu \nu}=J_{\mu} J_{\nu}^{*} .
$$

The leptonic tensor $L_{\mu \nu}$ for unpolarized initial and final electrons (averaging over the initial electron spin and summing over polarizations of the final electron) has the form

$$
L_{\mu \nu}=q^{2} g_{\mu \nu}+2\left(k_{1 \mu} k_{2 v}+k_{1 v} k_{2 \mu}\right) \text {. }
$$

The hadronic tensor $W_{\mu \nu}$ for unpolarized initial and final deuterons can be written in the standard form, in terms of two unpolarized structure functions:

$$
W_{\mu \nu}=\left(-g_{\mu \nu}+\frac{q_{\mu} q_{v}}{q^{2}}\right) W_{1}\left(q^{2}\right)+P_{\mu} P_{\nu} W_{2}\left(q^{2}\right),
$$

where $P_{\mu}=\left(p_{1}+p_{2}\right)_{\mu} /(2 M)$. Averaging over the spin of the initial deuteron, the structure functions $W_{i}\left(q^{2}\right), i=1,2$, can be expressed in terms of the electromagnetic form factors as

$$
\begin{aligned}
& W_{1}\left(q^{2}\right)=-\frac{2}{3} q^{2}(1+\tau) G_{M}^{2}\left(q^{2}\right), \\
& W_{2}\left(q^{2}\right)=4 M^{2}\left[G_{C}^{2}\left(q^{2}\right)+\frac{2}{3} \tau G_{M}^{2}\left(q^{2}\right)+\frac{8}{9} \tau^{2} G_{Q}^{2}\left(q^{2}\right)\right] .
\end{aligned}
$$

The expression of the differential cross section as a function of the recoil-electron energy, $\epsilon_{2}$, for unpolarized deuteronelectron scattering can be written as

$$
\frac{d \sigma^{(B)}}{d \epsilon_{2}}=\frac{\pi \alpha^{2}}{m|\vec{p}|^{2}} \frac{\mathcal{D}}{q^{4}},
$$

where $\vec{p}$ is the deuteron beam momentum and

$$
\begin{aligned}
\mathcal{D}= & \frac{1}{2} L_{\mu \nu} W_{\mu \nu} \\
= & 2\left[M^{2} q^{2}+2 m E\left(2 m E+q^{2}\right)\right]\left[G_{C}^{2}\left(q^{2}\right)+\frac{8}{9} \tau^{2} G_{Q}^{2}\left(q^{2}\right)\right] \\
& +\frac{4}{3} \tau\left[4 m^{2}\left(E^{2}-M^{2}\right)+q^{2}\left(m^{2}-M^{2}\right.\right. \\
& \left.\left.-2 \tau M^{2}+2 m E\right)\right] G_{M}^{2}\left(q^{2}\right) .
\end{aligned}
$$

This expression is valid in the one-photon exchange (Born) approximation in the reference system where the target electron is at rest.

The differential cross section, as a function of the fourmomentum transfer squared, is

$$
\frac{d \sigma^{(B)}}{d q^{2}}=\frac{\pi \alpha^{2}}{2 m^{2}|\vec{p}|^{2}} \frac{\mathcal{D}}{q^{4}} .
$$

Lastly, the differential cross section over the scatteredelectron solid angle has the following expression:

$$
\frac{d \sigma^{(B)}}{d \Omega_{e}}=\frac{\alpha^{2}}{8 m^{4}|\vec{p}|}\left(1-\frac{4 m^{2}}{q^{2}}\right)^{3 / 2} \frac{\mathcal{D}}{E+m} .
$$

\section{RADIATIVE CORRECTIONS}

Let us consider the QED radiative corrections which arise due to the soft- and hard-photon bremsstrahlung by the electrons, the electron vertex correction, and the electron vacuum polarization. The corresponding diagrams are shown in Fig. 1.

\section{A. Virtual and soft corrections}

In this section, we use the standard expressions for the UV finite parts of the electron vertex and the vacuum polarization as well soft-photon corrections written in the initial electron rest frame. The electron mass is always taken explicitly into account.

We use the Lorentz and gauge-invariant Pauli-Willars subtraction procedure [42] for the regularization of the ultraviolet (UV) divergent parts of the electron and photon self-energies 
and the electron vertex function. For the regularization of the infrared divergence, we introduce the auxiliary photon mass $\lambda$.

The UV divergent part of the photon self-energy (vacuum polarization) is included into the renormalization of the electric charge. The UV divergent part of the electron self-energy (mass operator)

$$
\begin{aligned}
& \Sigma(\hat{p})=\Sigma(m)+\left.\frac{\partial \Sigma(\hat{p})}{\partial \hat{p}}\right|_{\hat{p}=m}(\hat{p}-m)+C(\hat{p}), \\
& \Sigma(m)=\delta m,
\end{aligned}
$$

enters in the first two terms of its expansion by powers $(\hat{p}-m)$. For the electron mass, we choose its physical value. It means that the first term $\delta m$ in Eq. (17) is compensated by the counterterm in the Lagrangian. As concerns the second one, its contribution into the renormalization of the external electron wave function is exactly canceled by a particular UV regularization of the electron vertex correction:

$$
\begin{aligned}
\Lambda_{\mu}\left(p_{1}, p_{2}\right) & =\Lambda_{\mu}\left(p_{1}, p_{2}\right)-\Lambda_{\mu}(p, p)+\Lambda_{\mu}(p, p) \\
& =\bar{\Lambda}_{\mu}\left(p_{1}, p_{2}\right)+\Lambda_{\mu}(p, p) .
\end{aligned}
$$

The quantity $\bar{\Lambda}_{\mu}\left(p_{1}, p_{2}\right)$ is UV finite, and this cancellation takes place due to the Ward identity

$$
\Lambda_{\mu}(p, p)=-\partial \Sigma(\hat{p}) / \partial p_{\mu} .
$$

As the UV finite part of the mass operator $C(\hat{p})$ contains a term that is proportional to $(\hat{p}-m)^{2}$, the graphs corresponding to the electron self-energy on external electron lines do not contribute. This scheme of calculation is explained in Ref. [43], and it is gauge invariant. The Lorentz gauge is used here as well as in our previous paper [32].

The virtual and soft corrections are calculated by the standard method (see Ref. [44]) and the corresponding effect reads

$$
d \sigma^{(\mathrm{RC})}=\left(1+\delta_{0}+\bar{\delta}+\delta^{(\mathrm{vac})}\right) d \sigma^{(B)},
$$

with

$$
\begin{aligned}
\delta_{0}= & \frac{2 \alpha}{\pi} \ln \frac{\bar{\omega}}{m}\left[\frac{\epsilon_{2}}{\left|\vec{k}_{2}\right|} \ln \left(\frac{\epsilon_{2}+\left|\vec{k}_{2}\right|}{m}\right)-1\right], \\
\bar{\delta}= & \frac{\alpha}{\pi}\left(-1-2 \ln 2+\frac{\epsilon_{2}}{\left|\vec{k}_{2}\right|}\left\{\operatorname { l n } ( \frac { \epsilon _ { 2 } + | \vec { k } _ { 2 } | } { m } ) \left[1+\ln \left(\frac{\epsilon_{2}+\left|\vec{k}_{2}\right|}{m}\right)+2 \ln \left(\frac{m}{\left|\vec{k}_{2}\right|}\right)+\frac{m+3 \epsilon_{2}}{2 \epsilon_{2}}\right.\right.\right. \\
& \left.-\ln \left(\frac{\epsilon_{2}+m}{\left|\vec{k}_{2}\right|}\right)-\frac{1}{2} \ln \left(\frac{Q^{2}}{m^{2}}\right)\right]+4 m \frac{M^{2} q^{2}}{\epsilon_{2} \mathcal{D}}(1+\tau) \ln \left(\frac{\epsilon_{2}+\left|\vec{k}_{2}\right|}{m}\right)\left(G_{C}^{2}-\frac{4}{3} \tau G_{M}^{2}+\frac{8}{9} \tau^{2} G_{Q}^{2}\right) \\
& \left.\left.-\frac{\pi^{2}}{6}+L i_{2}\left(\frac{\epsilon_{2}-\left|\vec{k}_{2}\right|}{\epsilon_{2}+\left|\vec{k}_{2}\right|}\right)+L i_{2}\left(\frac{\epsilon_{2}+\left|\vec{k}_{2}\right|+m}{2\left(\epsilon_{2}+m\right)}\right)-L i_{2}\left(\frac{\epsilon_{2}-\left|\vec{k}_{2}\right|+m}{2\left(\epsilon_{2}+m\right)}\right)\right\}\right),
\end{aligned}
$$

where $\bar{\omega}$ is the maximal energy of the soft photon and we assume $\bar{\omega} \ll m$. Only the term containing the deuteron form factors in Eq. (22) differs from the case of elastic protonelectron scattering. It arises due to appearance of the additional structure $\sigma_{\mu \nu}$ in the electron vertex.

We separate the contribution $\delta_{0}$ since it can be summed at all orders of the perturbation theory (similarly to the exponentiation of the infrared factors arising from the real and virtual photons given in Ref. [45]) using the exponential form of the electron structure functions [46]. To do this, it is sufficient to keep only the exponential contributions in the electron structure functions. The final result can be obtained substituting the term $\left(1+\delta_{0}\right)$ by the following expression:

$$
\left(\frac{\bar{\omega}}{m}\right)^{\beta} \frac{\beta}{2} \int_{0}^{1} x^{\frac{\beta}{2}-1}(1-x)^{\frac{\beta}{2}} d x
$$

where, in accordance with our direct calculations,

$$
\beta=\frac{2 \alpha}{\pi}\left[\frac{\epsilon_{2}}{\left|\vec{k}_{2}\right|} \ln \left(\frac{\epsilon_{2}+\left|\vec{k}_{2}\right|}{m}\right)-1\right],
$$

and $\left(1+\delta_{0}\right)$ is the first two terms of the expansion (23) in powers of $\beta$.

The electron structure function method can be applied if the condition $Q^{2} \gg m^{2}$ is satisfied that is valid for the $Q^{2}$ values more than $10^{-5} \mathrm{GeV}^{2}$ (corresponding to $\epsilon_{2}>10 \mathrm{MeV}$ ). It is easy to verify that in this case

$$
\frac{\epsilon_{2}}{\left|\vec{k}_{2}\right|} \ln \left(\frac{\epsilon_{2}+\left|\vec{k}_{2}\right|}{m}\right)=\ln \frac{Q^{2}}{m^{2}}+O\left(\frac{m^{2}}{Q^{2}}\right) \text {. }
$$

\section{B. Hard-photon contribution}

In this section, we calculate the radiative correction due to the hard-photon bremsstrahlung

$$
d\left(p_{1}\right)+e\left(k_{1}\right) \rightarrow d\left(p_{2}\right)+e\left(k_{2}\right)+\gamma(k)
$$

with the photon energy $\omega>\bar{\omega}$ in the target electron rest frame.

In the experimental setup when only energies of the scattered deuteron and electron are measured, we use formalism developed in Ref. [40], where $\pi-e^{-}$scattering has been analyzed. We applied it to calculate the hard-photon correction in the elastic $p e$ scattering [32] and performing similar 


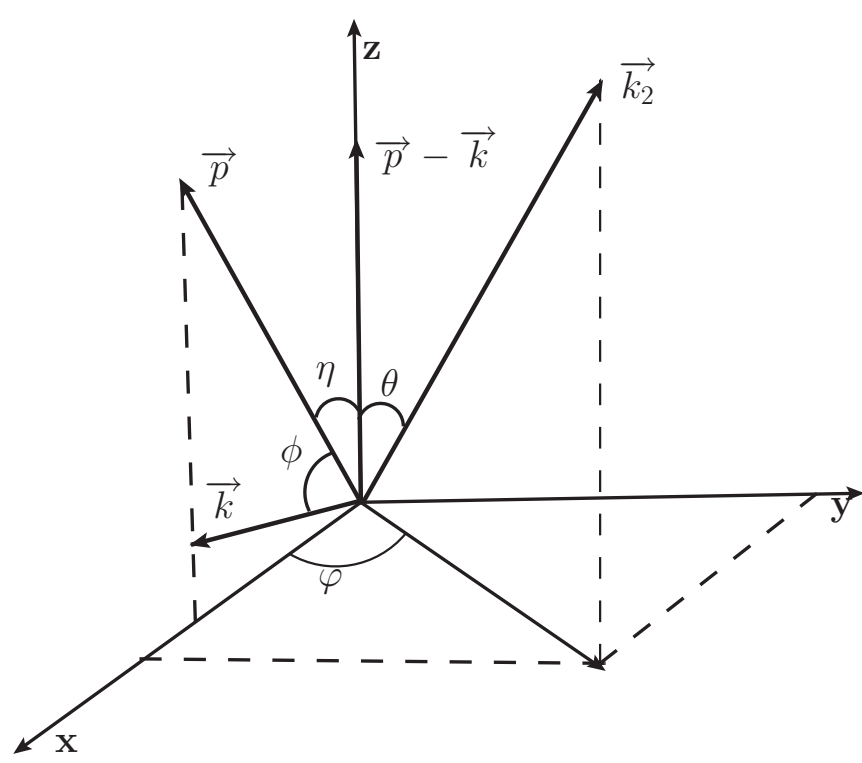

FIG. 2. Coordinate system and definition of the angles used for the integration over the variables of the final state.

calculations we derive for the cross section of the process (26) the following expression (averaging over the initial particle spins and summing over the polarizations of the final particles)

$$
\begin{aligned}
\frac{d \sigma^{(h)}}{d \epsilon_{2}}= & \frac{\alpha^{3}}{16 \pi} \frac{1}{m|\vec{p}|^{2}} \int \omega d \omega \int d y \int_{0}^{2 \pi} \frac{1}{q_{1}^{4}|\vec{p}-\vec{k}|} \\
& \times\left[-W_{1}\left(q_{1}^{2}\right) S_{1}+\frac{W_{2}\left(q_{1}^{2}\right)}{M^{2}} S_{2}\right] d \varphi .
\end{aligned}
$$

Here $q_{1}=k_{1}-k_{2}-k$ and $y=E-|\vec{p}| \cos \phi>0$. The coordinate system and the orientations of the three-momenta are shown in Fig. 2. The functions $S_{1,2}$ are expressed via the scalar products of the 4-momenta in the reaction (see Eqs. (40) and (41) in Ref. [32]) and the scalar products, in turn, are expressed via variables $\omega, y, \varphi^{1}$ and the initial deuteron 3 -momentum $|\vec{p}|$. The deuteron structure functions $W_{1}$ and $W_{2}$ are defined by relations (12) but now their argument is $q_{1}^{2}$.

Let us discuss the integration region in the right-hand side of Eq. (27). The events corresponding to the scattereddeuteron energy $E_{2} \pm \Delta E_{2}$ and the recoil-electron energy $\epsilon_{2} \pm \Delta \epsilon_{2}$ (they satisfy the condition $E+m=E_{2}+\epsilon_{2}$ ) are considered as true elastic events. Here, $\Delta E_{2}$ and $\Delta \epsilon_{2}$ are the uncertainties of the measurement of the final deuteron and recoil electron energies. The plot of the variable $E_{2}$ versus the variable $\epsilon_{2}$ is shown in Fig. 3. The shaded area in this figure represents the region where events are allowed by the experimental limitations. The relation between the energies $E_{2}$ and $\epsilon_{2}$, as shown in Fig. 3, has to be transformed into a limit on the possible photon momentum $\vec{k}$.

\footnotetext{
${ }^{1}$ There is a misprint in our paper [32]. The expression for $\cos \theta$ in the denominator of Eq. (49) has to be multiplied by the additional factor $\left|\vec{k}_{2}\right|$.
}

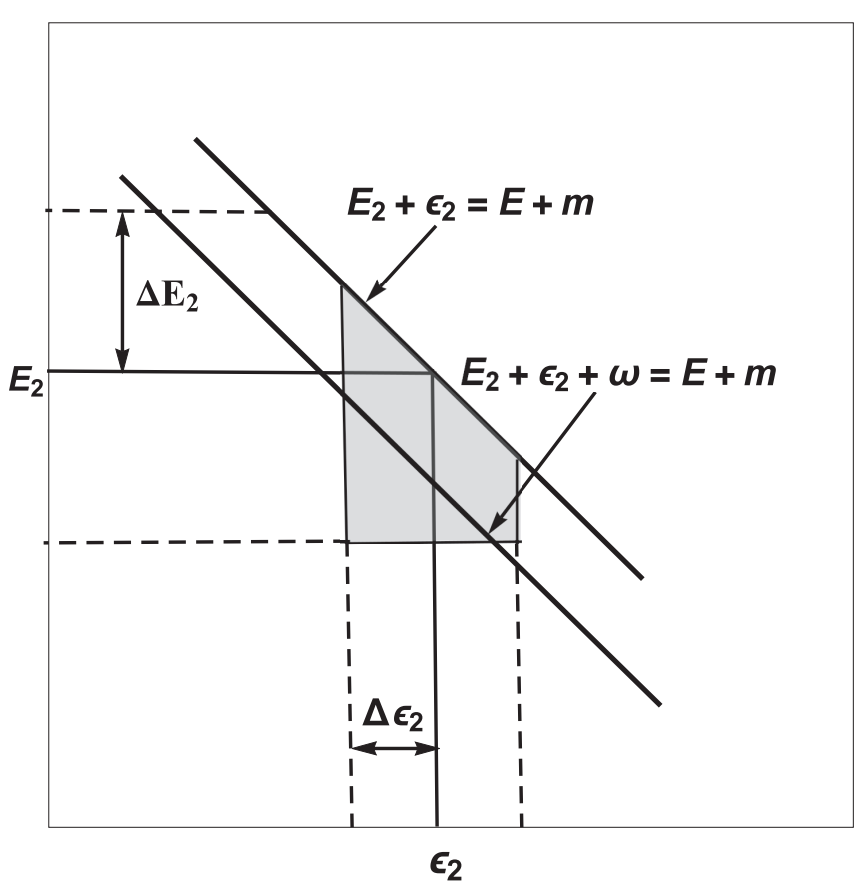

FIG. 3. The shaded area represents the kinematically allowed region within the experimental setup in the plane of the variables $E_{2}$ and $\epsilon_{2}$.

Usually the uncertainties $\Delta E_{2}$ and $\Delta \epsilon_{2}$ are proportional to $E_{2}$ and $\epsilon_{2}$, respectively. For deuteron beam energies up to $500 \mathrm{GeV}$, the recoil-electron energy is about two orders of magnitude smaller than the scattered deuteron one. Therefore, the following condition $\Delta E_{2} \gg \Delta \epsilon_{2}$ is satisfied and the effect due to the nonzero value of $\Delta \epsilon_{2}$ is negligible. Therefore, the numerical calculations are performed for $\Delta \epsilon_{2}=0$.

We consider the experimental setup where no angles are measured and therefore the orientation of the photon momentum $\vec{k}$ is not limited and we investigate both cases: (i) $\epsilon_{2}<$ $\epsilon_{2 \max }-\Delta E$ and (ii) $\epsilon_{2}>\epsilon_{2 \max }-\Delta E$, where $\Delta E=\Delta E_{2}$ and where $\epsilon_{2 \text { max }}$ is defined by Eq. (2).

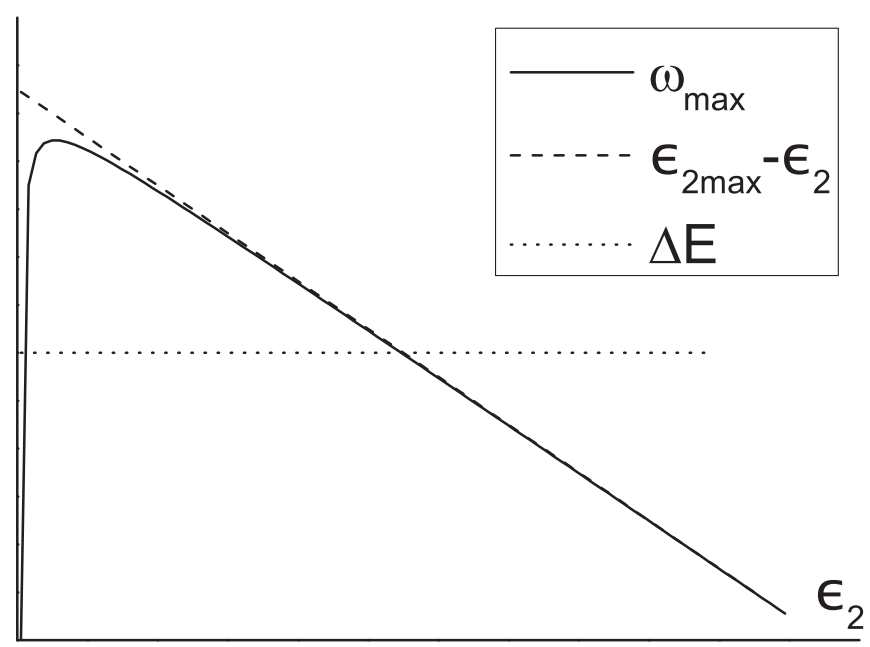

FIG. 4. Maximum energy of the photon, $\omega_{\max }$, in the case $\epsilon_{2}>\epsilon_{2 \max }-\Delta E$ as given by Eq. (29). 

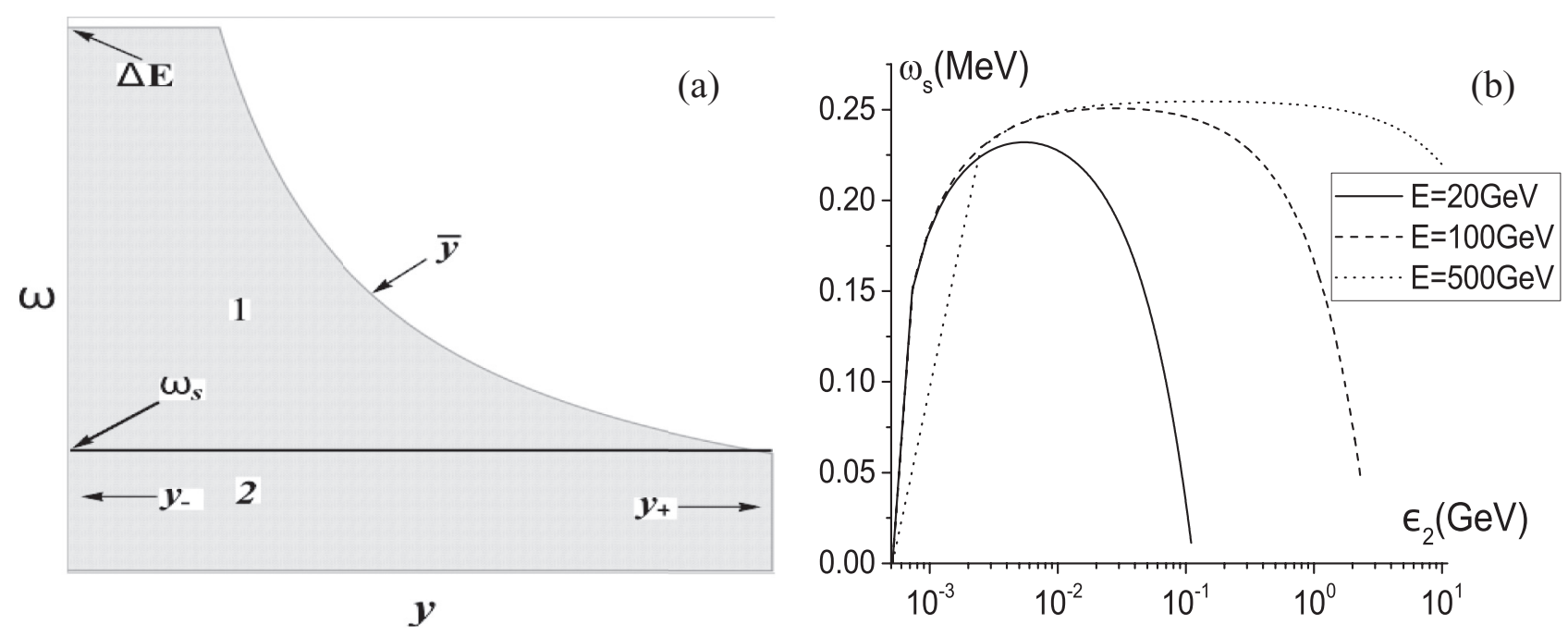

FIG. 5. (a) Integration region over the variables $\omega$ and $y$ in the case $\epsilon_{2}<\epsilon_{2 \max }-\Delta E$. Here $y_{ \pm}=E \pm p$. (b) $\omega_{s}$, defined as the positive solution of the equation $\bar{y}=y_{+}$and given by Eq. (45) in Ref. [32], as a function of the recoil-electron energy for the different deuteron energies.

In the first case, we get, as experimental limit, the isotropic condition $\omega \leqslant \Delta E$ (only this case was considered in our early paper [32]), whereas in the second case the upper limit of $\omega$ depends on the recoil-electron energy, $\omega \leqslant \omega_{\max }$, as shown in Fig. 4. The quantity $\omega_{\max }$ is the root of the equation $y_{-}=\bar{y}, y_{ \pm}=E \pm|\vec{p}|$ and $\bar{y}$ is, in turn, the positive solution of the equation $|\cos \theta|=1$ and reads

$$
\bar{y}=\frac{\left(m-\epsilon_{2}\right)\left(E-\epsilon_{2}-\omega\right)+\sqrt{\epsilon_{2}^{2}-m^{2}} \sqrt{\left(E+m-\epsilon_{2}-\omega\right)^{2}-M^{2}}}{\omega} .
$$

The quantity $\omega_{\max }$ has the following form:

$$
\begin{aligned}
\omega_{\max }= & \frac{\beta A}{B^{2}-C^{2}}, \quad \beta=2 m E+m^{2}+M^{2}, \\
A= & \left|\vec{k}_{2}\right|\left\{M^{2}\left[|\vec{p}|\left(E_{0}+|\vec{p}|\right)+\epsilon_{-}\left(E-m-2 \epsilon_{2}\right)\right]+2 m \epsilon_{-}\left(2 E E_{0}-m \epsilon_{-}\right)\right\} \\
& -\epsilon_{-}\left\{\left(E_{0}+|\vec{p}|\right)\left[4 m E E_{0}-2 m^{2} \epsilon_{-}+M^{2}\left(E-\epsilon_{2}\right)\right]-M^{2} E_{0} \epsilon_{+}\right\}, \\
B= & E\left(4 m^{2}+M^{2}\right)+m\left(2 E^{2}+2 m^{2}+M^{2}\right)-2 \beta \epsilon_{2}, \\
C= & |\vec{p}|\left(2 m E+M^{2}\right), \quad \epsilon_{ \pm}=\epsilon_{2} \pm m, E_{0}=E+m-\epsilon_{2} .
\end{aligned}
$$

The integration region over the variables $\omega$ and $y$ for the case $\omega \leqslant \Delta E$ is shown in Fig. 5(a), and the maximal photon energy $\omega_{s}$, when the photon can be emitted in the whole angular phase space (it means that $y_{-}<y<y_{+}$), is shown Fig. 5(b) (as a function of the recoil electron energy). The analytic form of $\omega_{s}$ is given by Eq. (45) of Ref. [32].

So, the integration region in (27) is divided in two parts, in the same way as for proton-electron scattering (see Eqs. (46) and (47) in Ref. [32]). However, as we take $\Delta \epsilon_{2}=0$, the weight function is always equal to 1 . In the limit $\omega \rightarrow 0$, the integrand in (27) has infrared behavior. The infrared contribution is combined with the corrections due to the soft-photon bremsstrahlung and the electron vertex. This results in the substitution $\bar{\omega} \rightarrow \omega_{s}$ in the expression for $\delta_{0}$; see Eq. (21). In the chosen experimental setup, the parameter $\bar{\omega}$ is nonphysical, and therefore it has to disappear in all the physical results. If the cancellation of this parameter is realized in the same way in all orders, we can include part of the hard-photon corrections which contains $\left[\beta \ln \left(\omega_{s} / m\right)\right]^{n}, n=1,2, \ldots$ in the exponential factor (23) by changing $\bar{\omega} \rightarrow \omega_{s}$. This can be verified by performing the direct calculations at least at the second order.

\section{NUMERICAL ESTIMATIONS AND DISCUSSION}

In this section, the conditions for the experimental uncertainties are set to $\Delta E=0.02\left(E-\epsilon_{2}\right)$ and the $t_{20}$ parametrization of the deuteron form factors is taken as below, unless otherwise specified.

In our calculation, we use four different parametrizations of the deuteron form factors, and since the four-momentum transfer squared is rather small in this reaction, we can approximate these form factors by a Taylor series expansion with a good accuracy. On the Born level and when calculating the soft-photon bremsstrahlung, electron vertex, and vacuum polarization contributions, we can use also unexpanded 
expressions, but, in order to perform the analytical integrations in Eqs. (27), we have to expand the differential cross section, keeping terms up to $q_{1}^{4}$ in $W_{1}\left(q_{1}^{2}\right)$ and $W_{2}\left(q_{1}^{2}\right)$.

Therefore, we use the expansion over the variable $q^{2}$ of the following four form factor parametrizations.

\section{A. By means of the radii (labeled as "rad")}

In this approach, we expand the quantity $\mathcal{D}$, which is defined by Eq. (14), including the terms up to $q^{4}$, and we use the expansion of the form factors, taking into account only the mean square charge and magnetic radii from Ref. [21]:

$$
\begin{aligned}
\frac{G_{C, M}\left(q^{2}\right)}{G_{C, M}(0)} & =1+\frac{1}{6} q^{2} r_{C, M}^{2}+O\left(q^{4}\right), \quad G_{Q}\left(q^{2}\right)=G_{Q}(0), \\
r_{C} & =2.130 \mathrm{fm}, \quad r_{M}=2.072 \mathrm{fm} .
\end{aligned}
$$

\section{B. Two-component model for the deuteron electromagnetic structure [47] (labeled as " $m$ ")}

In this approach, the deuteron form factors are saturated from the contribution of the isoscalar vector mesons, $\omega$ and $\phi$. In this case, one can write

$$
G_{i}\left(Q^{2}\right)=N_{i} g_{i}\left(Q^{2}\right) F_{i}\left(Q^{2}\right), \quad i=C, M, Q
$$

with

$$
F_{i}\left(Q^{2}\right)=1-\alpha_{i}-\beta_{i}+\alpha_{i} \frac{m_{\omega}^{2}}{m_{\omega}^{2}+Q^{2}}+\beta_{i} \frac{m_{\phi}^{2}}{m_{\phi}^{2}+Q^{2}},
$$

where $m_{\omega}\left(m_{\phi}\right)$ is the mass of the $\omega(\phi)$ meson. Note that the $Q^{2}$ dependence of $F_{i}\left(Q^{2}\right)$ is parameterized in such form that $F_{i}(0)=1$, for any values of the free parameters $\alpha_{i}$ and $\beta_{i}$, which are real numbers.

The terms $g_{i}\left(Q^{2}\right)$ are written as functions of two real parameters, $\gamma_{i}$ and $\delta_{i}$,

$$
g_{i}\left(Q^{2}\right)=1 /\left[1+\gamma_{i} Q^{2}\right]^{\delta_{i}},
$$

and $N_{i}$ is the normalization of the $i$ th form factor at $Q^{2}=0$ :

$$
\begin{aligned}
& N_{C}=G_{C}(0)=1, \quad N_{Q}=G_{Q}(0)=M^{2} \mathcal{Q}_{d}=25.83, \\
& N_{M}=G_{M}(0)=\frac{M}{m_{N}} \mu_{d}=1.714,
\end{aligned}
$$

where $\mathcal{Q}_{d}$ is expressed in $[\mathrm{GeV}]^{-2}$ units and $\mu_{D}$ is in nuclear magneton units.

The experimental data for $G_{C}$ and $G_{M}$ show the existence of a zero, for $Q_{0 C}^{2} \simeq 0.7 \mathrm{GeV}^{2}$ and $Q_{0 M}^{2} \simeq 2 \mathrm{GeV}^{2}$. The requirement of a node gives the following relation between the parameters $\alpha_{i}$ and $\beta_{i}, i=C$ and $M$ :

$$
\alpha_{i}=\frac{m_{\omega}^{2}+Q_{0 i}^{2}}{Q_{0 i}^{2}}-\beta_{i} \frac{m_{\omega}^{2}+Q_{0 i}^{2}}{m_{\phi}^{2}+Q_{0 i}^{2}} .
$$

The expression (31) contains four parameters, $\alpha_{i}, \beta_{i}, \gamma_{i}$, $\delta_{i}$, generally different for different form factors. It turns out that common values of the parameters $\delta=1.04 \pm 0.03$, $\gamma=12.1 \pm 0.5 \mathrm{GeV}^{-2}$ allow a good fit for all form factors, corresponding to $\chi^{2} / n d f=1.1$. The values of the best-fit parameters are reported in Table I. In our calculations, we used the central values of these parameters.
TABLE I. Values of the parameters $\alpha$ and $\beta$ for the three deuteron electromagnetic form factors, from the global fit. The parameters $\delta$ and $\gamma$ are common to all form factors. In the case of $G_{C}$ and $G_{M}, \alpha$ is derived from Eq. (34).

\begin{tabular}{cccc}
\hline \hline & $\alpha$ & $\beta$ & $\chi^{2} / n d f$ \\
\hline$G_{C}$ & $5.75 \pm 0.07$ & $-5.11 \pm 0.09$ & 0.9 \\
$G_{Q}$ & $4.21 \pm 0.05$ & $-3.41 \pm 0.07$ & 0.9 \\
$G_{M}$ & $3.77 \pm 0.04$ & $-2.86 \pm 0.05$ & 1.6 \\
\hline \hline
\end{tabular}

\section{Deuteron electromagnetic form factors in the transition region between nucleon-meson and quark-gluon pictures [48] (labeled as " $k$ ")}

In this approach, the deuteron form factors are consistent with the results from popular $N N$ potentials at low energies ( $Q^{2} \ll 1 \mathrm{GeV}^{2}$ ), but, at the same time, they provide the right asymptotic behavior following from the quark counting rules, at high energies $\left(Q^{2} \gg 1 \mathrm{GeV}^{2}\right)$. The explicit expressions of the deuteron form factors are

$$
\begin{aligned}
G_{C} & =\frac{G^{2}\left(Q^{2}\right)}{(2 \tau+1)}\left[\left(1-\frac{2}{3} \tau\right) A+\frac{8}{3} \sqrt{2 \tau} B+\frac{2}{3}(2 \tau-1) C\right] \\
G\left(Q^{2}\right) & =\left(1+\frac{Q^{2}}{4 \delta^{2}}\right)^{-2} \\
G_{M} & =\frac{G^{2}\left(Q^{2}\right)}{(2 \tau+1)}\left[2 A+\frac{2(2 \tau-1)}{\sqrt{2 \tau}} B-2 C\right] \\
G_{Q} & =\frac{G^{2}\left(Q^{2}\right)}{(2 \tau+1)}\left[-A+\sqrt{\frac{2}{\tau}} B-\frac{\tau+1}{\tau} C\right]
\end{aligned}
$$

where $\delta$ is some parameter of the order of the nucleon mass. The functions $A, B$, and $C$ have the following parametrizations,

$$
\begin{aligned}
& A=\sum_{i}^{n} \frac{a_{i}}{\alpha_{i}^{2}+Q^{2}}, \quad B=Q \sum_{i}^{n} \frac{b_{i}}{\beta_{i}^{2}+Q^{2}}, \\
& C=Q^{2} \sum_{i}^{n} \frac{c_{i}}{\gamma_{i}^{2}+Q^{2}},
\end{aligned}
$$

where $\left(a_{i}, \alpha_{i}\right),\left(b_{i}, \beta_{i}\right),\left(c_{i}, \gamma_{i}\right)$ are the fitting parameters. From the quark counting rules, it follows that the fall-off behavior of these amplitudes at high $Q^{2}$ is

$$
A \sim Q^{-2}, \quad B \sim Q^{-3}, \quad C \sim Q^{-4}
$$

which, together with the requirement of a correct static normalization, imposes the set of the constraints on $\left(a_{i}\right),\left(b_{i}\right),\left(c_{i}\right)$ :

$$
\begin{aligned}
\sum_{i}^{n} \frac{a_{i}}{\alpha_{i}^{2}} & =1, \quad \sum_{i}^{n} b_{i}=0, \quad \sum_{i}^{n} \frac{b_{i}}{\beta_{i}^{2}}=\frac{2-\mu_{d}}{2 \sqrt{2} M}, \\
\sum_{i}^{n} c_{i} & =0, \quad \sum_{i}^{n} c_{i} \gamma_{i}^{2}=0, \quad \sum_{i}^{n} \frac{c_{i}}{\gamma_{i}^{2}}=\frac{1-\mu_{d}-M^{2} \mathcal{Q}_{d}}{4 M^{2}} .
\end{aligned}
$$


TABLE II. Parameters in Eq. (37) for $n=4$, see also Eq. (42) in Ref. [48].

\begin{tabular}{lcclc}
\hline \hline$i$ & 1 & 2 & \multicolumn{1}{c}{3} & \multicolumn{1}{c}{4} \\
\hline$a_{i} \mathrm{fm}^{-2}$ & 2.4818 & -10.850 & 6.4416 & Eq. (42) [48] \\
$b_{i} \mathrm{fm}^{-1}$ & -1.7654 & 6.7874 & Eq. (42) [48] & Eq. (42) [48] \\
$c_{i}$ & -0.053830 & Eq. (42) [48] & Eq. (42) [48] & Eq. (42) [48] \\
& $\alpha_{1}^{2}=1.8591 \mathrm{fm}^{-2}$ & $\mu^{(\alpha)}=0.58327 \mathrm{GeV}$ \\
& $\beta_{1}^{2}=19.586 \mathrm{fm}^{-2}$ & $\mu^{(\beta)}=0.1 \mathrm{GeV}$ \\
& $\gamma_{1}^{2}=1.0203 \mathrm{fm}^{-2}$ & $\mu^{(\gamma)}=0.17338 \mathrm{GeV}$ \\
& $\delta=0.89852 \mathrm{GeV}$ \\
\hline \hline
\end{tabular}

In our calculations, we used the following sequence for each group of these parameters:

$$
\begin{aligned}
& \alpha_{n}^{2}=2 M \mu^{(\alpha)} \\
& \alpha_{i}^{2}=\alpha_{1}^{2}+\frac{\alpha_{n}^{2}-\alpha_{1}^{2}}{n-1}(i-1), \quad i=1, \ldots, n
\end{aligned}
$$

(similarly, for $\beta_{i}$ and $\gamma_{i}$ ), where $\mu^{(\alpha)}, \mu^{(\beta)}$, and $\mu^{(\gamma)}$ have the dimension of energy. The parameters are listed in Table II for $n=4$.

\section{Jefferson $t_{20}$ Collaboration (labeled as $t_{20}$ )}

The three deuteron electromagnetic form factors have been determined by fitting directly the all existing measured differential cross section and polarization observables, according to the following expressions [49]:

$$
\begin{aligned}
G_{i}\left(Q^{2}\right) & =G_{i}(0) D_{i}\left(Q^{2}\right) I_{i}\left(Q^{2}\right), \quad D_{i}\left(Q^{2}\right)=1-\frac{Q^{2}}{Q_{i}^{2}}, \\
I_{i}\left(Q^{2}\right) & =\frac{1}{1+S_{i}\left(Q^{2}\right)}, \quad i=C, M, Q
\end{aligned}
$$

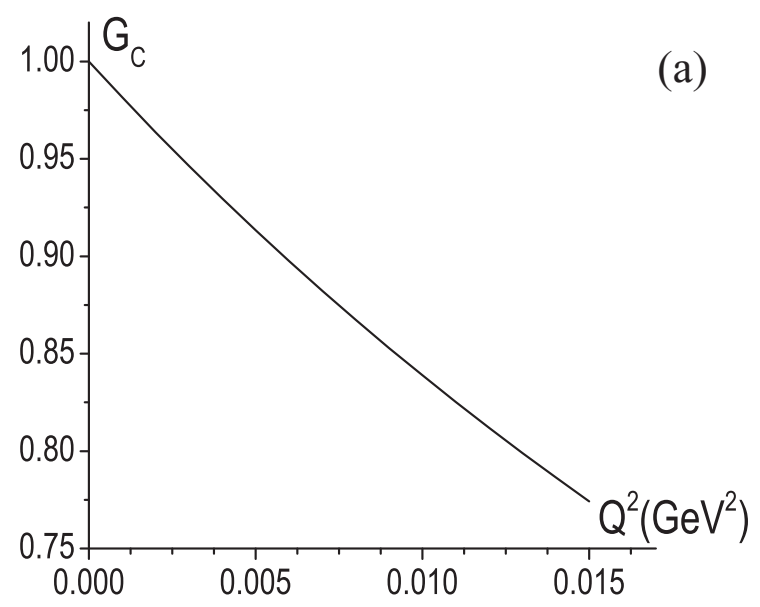

TABLE III. Parameters corresponding to Eq. (39) in $\mathrm{fm}^{2}$ units.

\begin{tabular}{lccrrr}
\hline \hline$k$ & 1 & 2 & 3 & 4 & \multicolumn{1}{c}{5} \\
\hline$a_{C}$ & 0.674 & 0.02246 & 0.009806 & -0.0002709 & 0.000003793 \\
$a_{M}$ & 0.5804 & 0.08701 & -0.003624 & 0.0003448 & -0.000002818 \\
$a_{Q}$ & 0.8796 & -0.5656 & 0.01933 & -0.0006734 & 0.000009438 \\
\hline \hline
\end{tabular}

where

$$
\begin{aligned}
& S_{i}\left(Q^{2}\right)=\sum_{k=1}^{5} a_{i}^{k} Q^{2 k}, \quad Q_{C}^{2}=17.72 \mathrm{fm}^{-2}, \\
& Q_{M}^{2}=54.32 \mathrm{fm}^{-2}, \quad Q_{Q}^{2}=65.61 \mathrm{fm}^{-2} \text {. }
\end{aligned}
$$

The parameters $a_{i}^{k}$ have dimensions of inverse $Q^{2}$ powers so that the quantities $S_{i}\left(Q^{2}\right)$ are dimensionless. The values of these parameters are shown in Table III with $Q^{2}$ in units of $\mathrm{fm}^{-2}$.

In our calculations, we restrict ourselves to values of $Q^{2}$ below $0.015 \mathrm{GeV}^{2}$. The most important contribution in this region is given by the deuteron charge form factor $G_{C}\left(Q^{2}\right)$. Its behavior is shown in Fig. 6(a) for the $t_{20}$ parametrization. Figure 6(b) shows a comparison among the parametrization in terms of the quantities $\Delta G_{C}^{i}$ defined as

$$
\Delta G_{C}^{i}=100\left[1-\frac{G_{C}^{i}\left(Q^{2}\right)}{G_{C}^{20}\left(Q^{2}\right)}\right], \quad i=m, k, \operatorname{rad} .
$$

To illustrate the dependence of the recoil-electron distribution on the deuteron beam energy, the Born cross section is shown in Fig. 7 for the standard $t_{20}$ parametrization at $E=20$, 100 , and $500 \mathrm{GeV}$. Here and below, for the beam energy $500 \mathrm{GeV}$, we limit the recoil-electron energy to $10 \mathrm{GeV}$, because for the largest values the expansions for the form factors are incorrect.

FIG. 6. (a) $Q^{2}$ dependence of the deuteron charge form factor for the $t_{20}$ parametrization and (b) of the quantities $\Delta G_{C}^{i}, i=m, k$, rad, as defined in the text. 


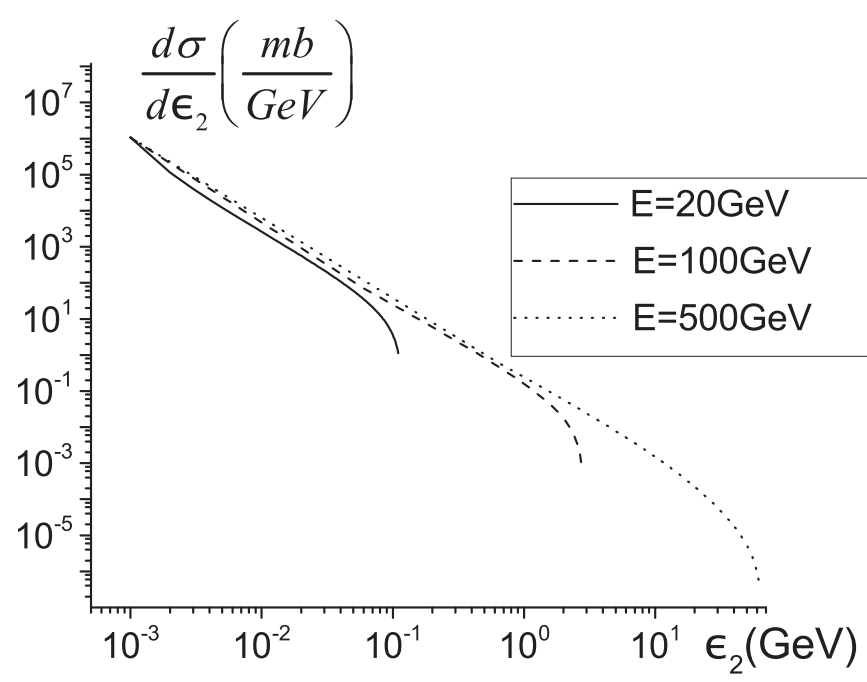

FIG. 7. Born differential cross section, defined by Eq. (13), calculated with the $t_{20}$ parametrization of the form factors, at different beam energies.
The sensitivity (in percent) of this cross section to different form factor parametrizations is shown in Fig. 8, in terms of the quantities

$$
R^{k}=1-\frac{d \sigma^{k}}{d \sigma^{t_{20}}}, \quad R^{m}=1-\frac{d \sigma^{m}}{d \sigma^{t_{20}}}, \quad R^{\mathrm{rad}}=1-\frac{d \sigma^{\mathrm{rad}}}{d \sigma^{t_{20}}}
$$

where $d \sigma^{i}$ is the differential cross section (13), and $i=$ $k, m, r a d, t_{20}$ correspond to the above-mentioned deuteron form factors. As one can see, the sensitivity has a very similar behavior for the expanded and unexpanded cross sections and increases when both the deuteron and recoil-electron energies increase. However, the differential cross section decreases very quickly when the recoil-electron energy increases (see Fig. 7).

The hard-photon correction depends on the parameter $\Delta E$ due to the contribution of the region indicated 1 in Fig. 5(a). To illustrate this dependence, we show in Fig. 9 the quantity (in which the contribution of the region 2 is removed)

$$
\Delta h=\frac{d \sigma^{(h)}\left[\Delta E=0.05\left(E_{2}-\epsilon_{2}\right)\right]-d \sigma^{(h)}\left[\Delta E=c_{i}\left(E_{2}-\epsilon_{2}\right)\right]}{d \sigma^{(B)}}, \quad c_{1}=0.005, \quad c_{2}=0.01, \quad c_{3}=0.02
$$

as a function of the recoil-electron energy for the $t_{20}$ parametrization. The effect is rather small: of the order of $1 \%(0.1 \%)$ for $E=500(100) \mathrm{GeV}$. For deuteron energy equal to $500 \mathrm{GeV}$ [Figs. 9(d)-9(f)], this dependence exhibits a monotonic increase with the recoil-electron energy, whereas at the energy $100 \mathrm{GeV}$ [Figs. 9(a)-9(c)], it has maximum and
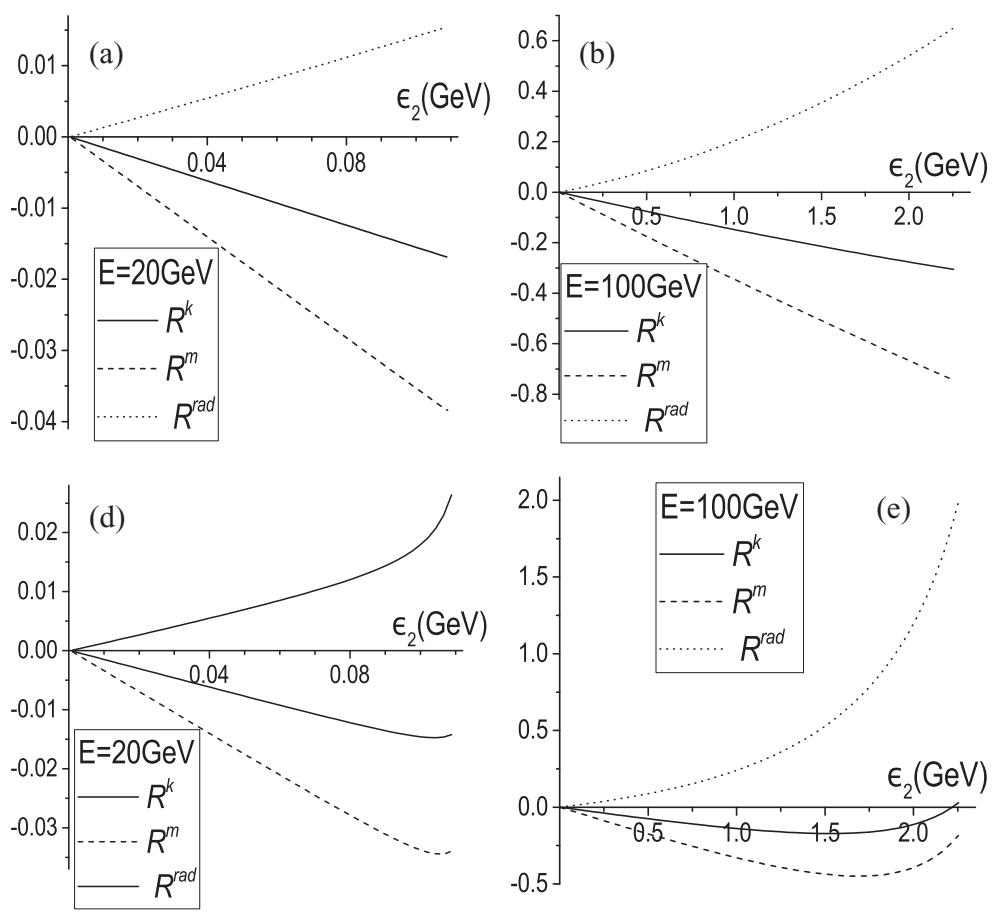
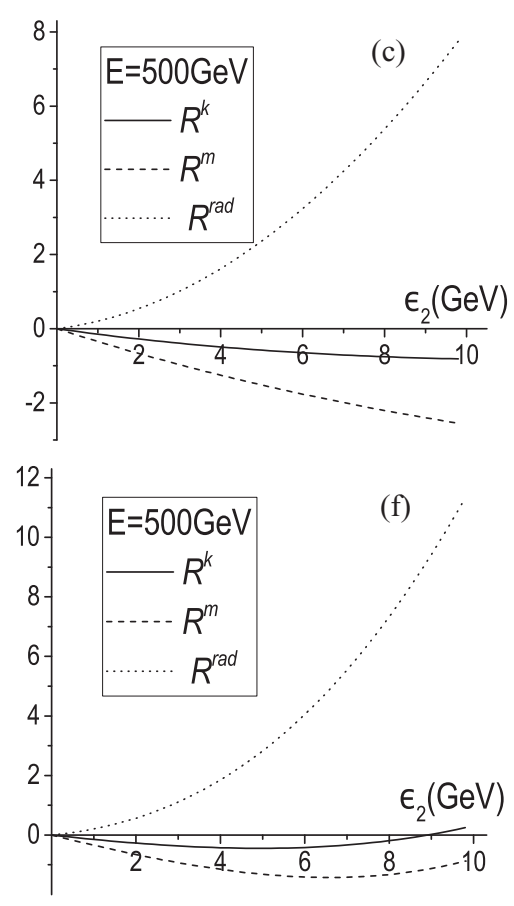

FIG. 8. Difference of the recoil-electron distributions, Eq. (42), in percent, normalized to $d \sigma^{t_{20}}$, for various parametrizations of the form factors, at deuteron energies $20 \mathrm{GeV}$ [(a), (d)], $100 \mathrm{GeV}$ [(b), (e)], and $500 \mathrm{GeV}$ [(c), (f)]. The upper set (a), (b), and (c) corresponds to the unexpanded form factors and the lower one (d), (e), and (f) corresponds to the expanded form factors, keeping the terms up to $q^{4}$. 

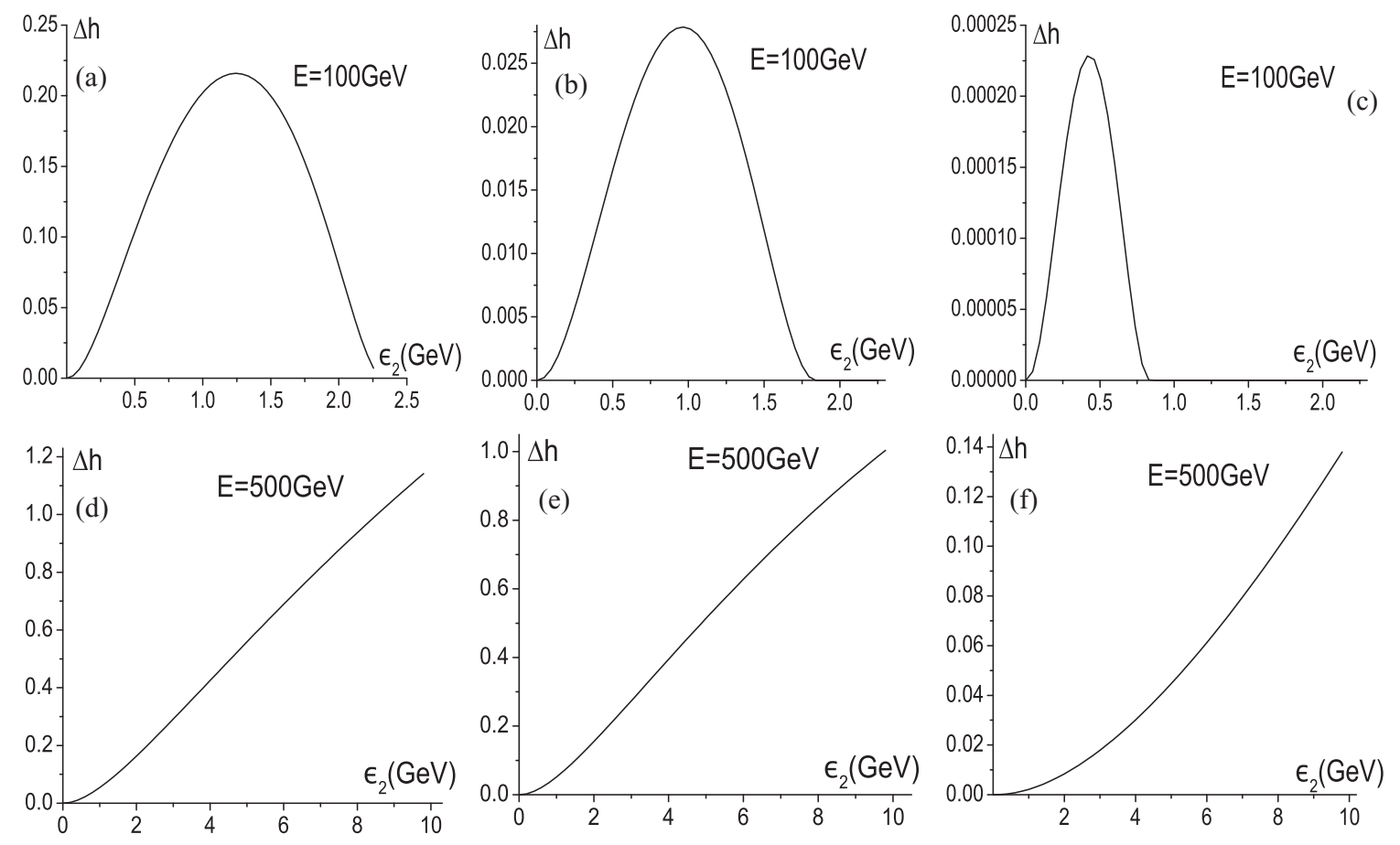

FIG. 9. The quantity $\Delta h$ (in percent) calculated according to Eq. (43), is shown as a function of the recoil-electron energy, at the deuteron energy of $100 \mathrm{GeV}$ [(a), (b), (c)] and $500 \mathrm{GeV}$ [(d), (e), (f)]. The left panels, (a) and (d), correspond to $c=0.005$, the middle ones, (b) and (e), correspond to $c=0.01$, and the right ones, (c) and (f), correspond to $c=0.02$.

then decreases down to zero. At this zero point, the value of the recoil-electron energy is the root of the equation $c_{i}\left(E_{2}-\right.$ $\left.\epsilon_{2}\right)=\omega_{\max }\left(\epsilon_{2}\right)$, provided that the line $\Delta E$ in Fig. 4 lies above the curve $\omega_{\max }\left(\epsilon_{2}\right)$. For the deuteron energy $100 \mathrm{GeV}$ and $\Delta E=0.05\left(E-\epsilon_{2}\right)$, this last condition is satisfied for all possible values of the recoil-electron energy, whereas for the energy $500 \mathrm{GeV}$ it is not fulfilled.

The bell form of the curves in Figs. 9(a)-9(c) is the interplay of two factors that give opposite effects when the recoilelectron energy grows. The first factor is $\Delta E$ independent and increases the ratio $d \sigma^{(h)} / d \sigma^{(B)}$ from its zero value at low $\epsilon_{2}$. However, the energy phase space of the photon becomes $\Delta E$ independent at large values of $\epsilon_{2}$ (see Fig. 4). As, for every value of $\epsilon_{2}$, the first term in the numerator of the Eq. (43) is greater than or equal to the second one, the quantity $\Delta h$ must have a maximum.

Figure 10 shows the quantities $\delta^{(h)}$ and $\widetilde{\delta}$, defined as

$$
\begin{aligned}
& \delta^{(h)}=\frac{d \sigma^{(h)}}{d \sigma^{(B)}}-\frac{2 \alpha}{\pi} \ln \frac{\omega_{s}}{\bar{\omega}}\left[\frac{\epsilon_{2}}{\left|\vec{k}_{2}\right|} \ln \left(\frac{\epsilon_{2}+\left|\vec{k}_{2}\right|}{m}\right)-1\right], \\
& \widetilde{\delta}=\bar{\delta}+\delta^{(\mathrm{vac})}+\frac{2 \alpha}{\pi} \ln \frac{\omega_{s}}{m}\left[\frac{\epsilon_{2}}{\left|\vec{k}_{2}\right|} \ln \left(\frac{\epsilon_{2}+\left|\vec{k}_{2}\right|}{m}\right)-1\right],
\end{aligned}
$$

and called, respectively, modified hard and soft and virtual corrections, as well as their sum $\delta_{\text {tot }}=\delta^{(h)}+\widetilde{\delta}$. This is the total first-order leptonic radiative correction, i.e. (the last term in $\tilde{\delta}$ is $\left.\delta_{0}\left(\bar{\omega} \rightarrow \omega_{s}\right)\right)$,

$$
\delta_{\mathrm{tot}}=\delta^{(h)}+\tilde{\delta}=\delta_{0}+\bar{\delta}+\delta^{(\mathrm{vac})}+\frac{d \sigma^{(h)}}{d \sigma^{(B)}} .
$$

Note that both modified corrections in Eq. (44) are independent on the auxiliary parameter $\bar{\omega}$ but depend on the physical parameter $\omega_{s}$ and therefore have a physical meaning.

To calculate $\delta_{\text {tot }}$, we can write the quantity $\left[1+\delta_{0}(\bar{\omega} \rightarrow\right.$ $\left.\omega_{s}\right)$ ] using the expressions (21) and (22) or its exponential form defined by (23) (with the substitution $\bar{\omega} \rightarrow \omega_{s}$ ). But numerical estimations show that they differ very little, by a few tenths of the percent, and therefore further we do not use the exponential form.

At small values of the squared momentum transfer (small recoil electron energy $\epsilon_{2}$ ), the total radiative correction is positive and decreases with increase of $\epsilon_{2}$, reaching zero and becoming negative. The absolute value of the radiative correction does not exceed 6\%, although a strong compensation of the large (up to $30 \%$ ) positive modified hard and negative modified soft and virtual corrections takes place. Such behavior of the pure QED correction is similar to one derived in Ref. [40].

If the deuteron form factors are determined independently with high accuracy from other experiments, the measurement of the cross section $d \sigma / d \epsilon_{2}$ can be used, in principle, to measure the hadronic part of the radiative correction in the considered conditions. This possibility is similar to the one described in Ref. [50], where the authors proposed to determine the hadronic (model-dependent) contribution to the running electromagnetic coupling $\alpha\left(q^{2}\right)$ by a precise measurement 

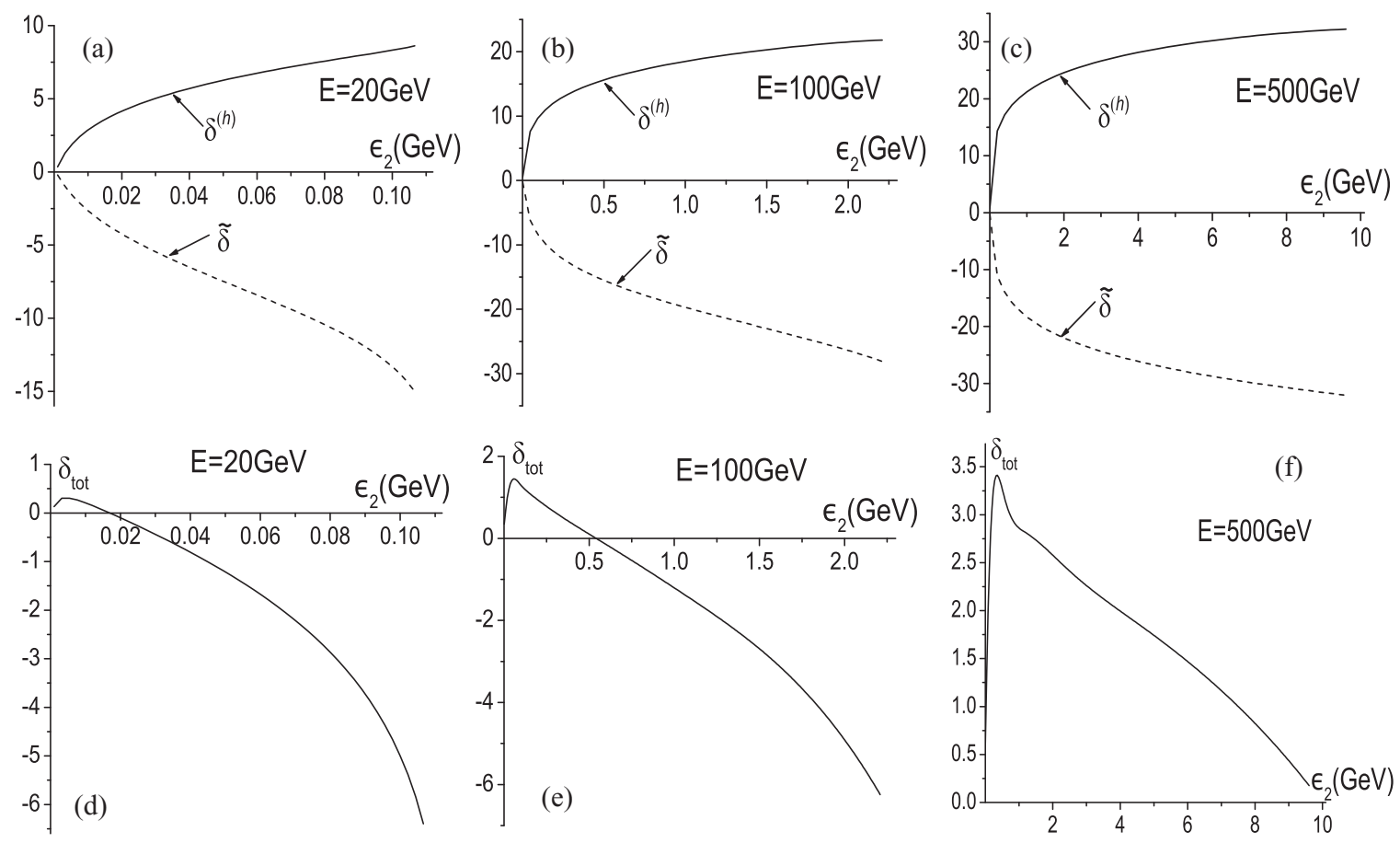

FIG. 10. Top: modified soft and virtual $(\tilde{\delta})$ (dashed line) and hard $\left(\delta^{(h)}\right)$ (solid line) corrections in percent as defined in Eq. (44) at incident deuteron energies $E=20 \mathrm{GeV}$ (a), $100 \mathrm{GeV}$ (b), and $500 \mathrm{GeV}$ (c). Bottom: total radiative correction (in percent) calculated for the standard $t_{20}$ fit for $\Delta E=0.02\left(E-\epsilon_{2}\right)$, and $E=20 \mathrm{GeV}$ (d), $100 \mathrm{GeV}$ (e), and $500 \mathrm{GeV}$ (f).

of the $\mu^{-} e^{-}$differential cross section, assuming that QED model-independent radiative corrections are under control.

In Fig. 11, we illustrate the sensitivity of the total radiative correction to the parametrization of the form factors in terms of the ratios

$$
P^{i}=\frac{1+\delta_{\mathrm{tot}}^{i}}{1+\delta_{\mathrm{tot}}}-1, \quad i=k, m, \mathrm{rad},
$$

where $\delta_{\text {tot }}$ is the total correction for the standard $t_{20}$ fit. We see that, in the considered conditions, the deviation of these quantities from unity is very small. We conclude that the influence of the parametrizations of the form factors on the radiative corrections is much smaller than on the Born cross section.

\section{CONCLUSION}

In this paper, we studied deuteron elastic scattering on electron at rest. We derived the differential cross section including QED radiative corrections to the leptonic part of the interaction, in the case of a coincidence experimental setup. The recoil-electron energy distribution in elastic deuteron-electron scattering is illustrated. The detection of the recoil electron, in the energy range from a few $\mathrm{MeV}$ up to $10 \mathrm{GeV}$, allows us to collect small- $Q^{2}$ data, at $10^{-5} \mathrm{GeV}^{2} \leqslant Q^{2} \leqslant 10^{-2} \mathrm{GeV}^{2}$. Such data, combined with the existing and future experiments with electron beams, will bring new information on the small$Q^{2}$ behavior of the deuteron electromagnetic form factors. This allows us to further constrain the extrapolation to the static point for the extraction of the deuteron charge radius.
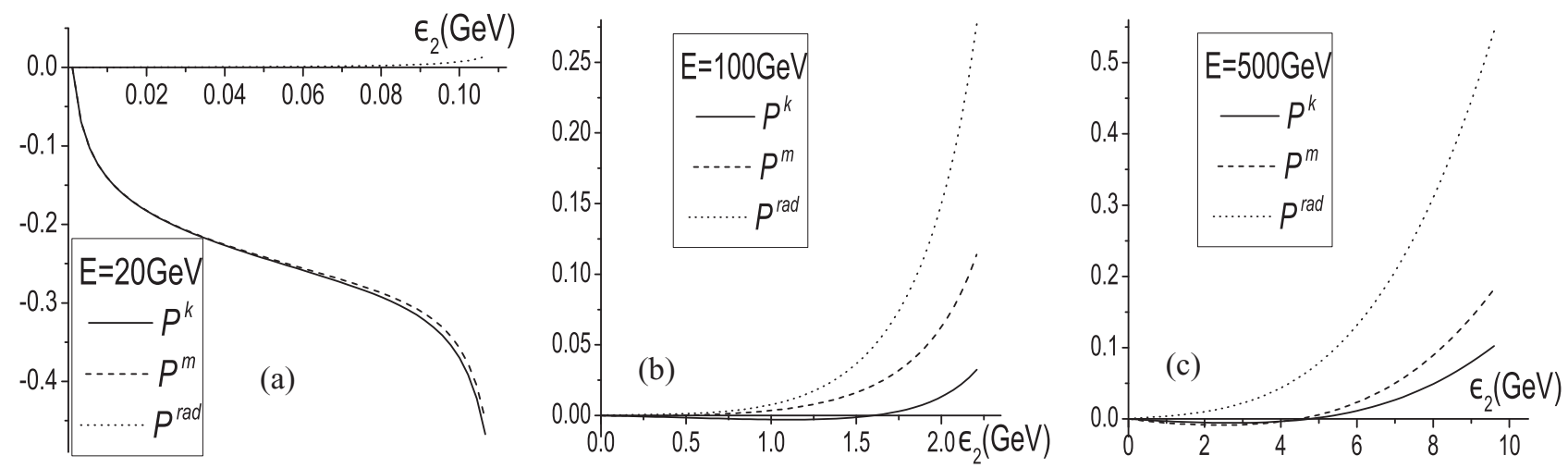

FIG. 11. Sensitivity of the total radiative corrections in percent to the choice of the form factor parametrization, Eq. (46), for different deuteron energies: $E=20 \mathrm{GeV}$ (a), $100 \mathrm{GeV}$ (b), and $500 \mathrm{GeV}$ (c). 
The measurements in the region of small- $Q^{2}$ values require high-energy deuteron beams, of the order of a few hundreds $\mathrm{GeV}$. The sensitivity of the differential cross section to the form factors parametrizations, labeled as $k, m$, and $t_{20}$, is small (does not exceed 2\%), but the rad parametrization gives a value of the cross section about $10 \%$ smaller as compared with the $t_{20}$ parametrization at $Q^{2} \approx 10^{-2} \mathrm{GeV}^{2}$ (see Fig. 8).

The nontrivial part of the radiative corrections is the hardphoton bremsstrahlung contribution which takes place due to the uncertainty in the measurement of the deuteron (electron) energy, $\Delta E_{2}\left(\Delta \varepsilon_{2}\right)$. In our calculations, we follow Ref. [40] for the choice of the coordinate system and the angular integration method. To obtain the recoil-electron energy distribution, the integration in Eq. (27) over variables $\varphi$ and $y$ is performed analytically and the remaining $\omega$ integration is done numerically.

We assumed that uncertainties in the final particle energies are proportional to their energies and we showed that the effect due to the nonzero quantity $\Delta \varepsilon_{2}$ is negligible.

The total correction $\left|\delta_{\text {tot }}\right|$ does not exceed $6 \%$ at $E=$ $20 \mathrm{GeV}$ and $100 \mathrm{GeV}$ and $3.5 \%$ at $E=500 \mathrm{GeV}$ for the value $\Delta E_{2}=0.02\left(E-\epsilon_{2}\right)$ and the $t_{20}$ parametrization used in these calculations. The total correction shows a weak dependence on the form factor parametrization in the considered region (see. Fig. 11). At the lower values of $Q^{2}$, which correspond to the lower values of the recoil electron energy $\varepsilon_{2}$, the total correction $\delta_{\text {tot }}$ is positive and changes sign when $Q^{2}$ increases. Such behavior of $\delta_{\text {tot }}$ is similar to that found in Ref. [40] and confirmed in Ref. [51] for the case of pion-electron scattering.

In our paper, we do not consider the radiative corrections involving deuteron as well as the background due to the Coulomb and strong interactions between deuteron and atomic nuclei. These questions require additional detailed investigations due to the particular kinematical conditions and will be object of a different work.

\section{ACKNOWLEDGMENTS}

This work was partially supported by the Ministry of Education and Science of Ukraine (Projects No. 0115U000474 and No. 0117U004866), and by the National Academy of Sciences of Ukraine (Project No. Ts-3/53-2018). The research is carried on in the frame of the France-Ukraine IDEATE International Associated Laboratory (LIA). Thanks are due to Nancy Paul-Hupin for a careful reading of the paper.
[1] S. Pacetti, R. Baldini Ferroli, and E. Tomasi-Gustafsson, Phys. Rep. 550-551, 1 (2015).

[2] V. Punjabi, C. F. Perdrisat, M. K. Jones, E. J. Brash, and C. E. Carlson, Eur. Phys. J. A 51, 79 (2015).

[3] A. Akhiezer and M. Rekalo, Sov. Phys. Dokl. 13, 572 (1968) [Sov. J. Part. Nucl. 4, 277 (1974)].

[4] A. J. R. Puckett, E. J. Brash, M. K. Jones, W. Luo, M. Meziane, L. Pentchev, C. F. Perdrisat, V. Punjabi, F. R. Wesselmann, A. Afanasev et al., Phys. Rev. C 96, 055203 (2017).

[5] A. Afanasev, P. G. Blunden, D. Hasell, and B. A. Raue, Prog. Part. Nucl. Phys. 95, 245 (2017).

[6] C. E. Carlson and M. Vanderhaeghen, Annu. Rev. Nucl. Part. Sci. 57, 171 (2007).

[7] J. Arrington, P. G. Blunden, and W. Melnitchouk, Prog. Part. Nucl. Phys. 66, 782 (2011).

[8] P. G. Blunden, W. Melnitchouk, and J. A. Tjon, Phys. Rev. Lett. 91, 142304 (2003).

[9] P. A. M. Guichon and M. Vanderhaeghen, Phys. Rev. Lett. 91, 142303 (2003).

[10] I. A. Rachek, J. Arrington, V. F. Dmitriev, V. V. Gauzshtein, R. E. Gerasimov, A. V. Gramolin, R. J. Holt, V. V. Kaminskiy, B. A. Lazarenko, S. I. Mishnev et al., Phys. Rev. Lett. 114, 062005 (2015).

[11] B. S. Henderson, L. D. Ice, D. Khaneft, C. O'Connor, R. Russell, A. Schmidt, J. C. Bernauer, M. Kohl, N. Akopov, R. Alarcon et al. (OLYMPUS Collaboration), Phys. Rev. Lett. 118, 092501 (2017).

[12] D. Rimal, D. Adikaram, B. A. Raue, L. B. Weinstein, J. Arrington, W. K. Brooks, M. Ungaro, K. P. Adhikari, A. V. Afanasev, Z. Akbar et al. (CLAS Collaboration), Phys. Rev. C 95, 065201 (2017).

[13] M. P. Rekalo, E. Tomasi-Gustafsson, and D. Prout, Phys. Rev. C 60, 042202 (1999).
[14] E. A. Kuraev, M. Shatnev, and E. Tomasi-Gustafsson, Phys. Rev. C 80, 018201 (2009).

[15] R. Pohl, A. Antognini, F. Nez, F. D. Amaro, F. Biraben, J. M. R. Cardoso, D. S. Covita, A. Dax, S. Dhawan, L. M. P. Fernandes et al., Nature (London) 466, 213 (2010).

[16] A. Antognini, F. Nez, K. Schuhmann, F. D. Amaro, F. Biraben, J. M. R. Cardoso, D. S. Covita, A. Dax, S. Dhawan, M. Diepold et al., Science 339, 417 (2013).

[17] P. J. Mohr, D. B. Newell, and B. N. Taylor, Rev. Mod. Phys. 88, 035009 (2016).

[18] A. Antognini, F. D. Amaro, F. Biraben, J. M. R. Cardoso, D. S. Covita, A. Dax, S. Dhawan, L. M. P. Fernandes, A. Giesen, T. Graf et al., J. Phys.: Conf. Ser. 312, 032002 (2011).

[19] C. E. Carlson, Prog. Part. Nucl. Phys. 82, 59 (2015).

[20] R. J. Hill, EPJ Web Conf. 137, 01023 (2017).

[21] I. Sick, Prog. Part. Nucl. Phys. 47, 245 (2001).

[22] R. A. Gilman and F. Gross, J. Phys. G 28, R37 (2002).

[23] F. Gross, Eur. Phys. J. A 17, 407 (2003).

[24] C. Liang, Y. Dong, and W. Liang, Commun. Theor. Phys. 62, 383 (2014).

[25] N. G. Kelkar and D. Bedoya Fierro, Phys. Lett. B 772, 159 (2017).

[26] R. Pohl, F. Nez, L. M. P. Fernandes, F. D. Amaro, F. Biraben, J. M. R. Cardoso, D. S. Covita, A. Dax, S. Dhawan, M. Diepold et al. (CREMA Collaboration), Science 353, 669 (2016)

[27] P. J. Mohr, B. N. Taylor, and D. B. Newell, Rev. Mod. Phys. 84, 1527 (2012).

[28] R. Pohl, F. Nez, T. Udem, A. Antognini, A. Beyer, H. Fleurbaey, A. Grinin, T. W. Hänsch, L. Julien, F. Kottmann et al., Metrologia 54, L1 (2017).

[29] G. Gakh, A. Dbeyssi, E. Tomasi-Gustafsson, D. Marchand, and V. Bytev, Phys. Part. Nucl. Lett. 10, 393 (2013). 
[30] I. V. Glavanakov, Y. F. Krechetov, A. P. Potylitsyn, G. M. Radutsky, A. N. Tabachenko, and S. B. Nurushev, Nucl. Instrum. Methods A 381, 275 (1996); I. V. Glavanakov, Y. F. Krechetov, G. M. Radutskii, and A. N. Tabachenko, JETP Lett. 65, 131 (1997) [Pisma Zh. Eksp. Teor. Fiz. 65, 123 (1997)].

[31] G. I. Gakh, A. Dbeyssi, D. Marchand, E. Tomasi-Gustafsson, and V. V. Bytev, Phys. Rev. C 84, 015212 (2011).

[32] G. I. Gakh, M. I. Konchatnij, N. P. Merenkov, and E. TomasiGustafsson, Phys. Rev. C 95, 055207 (2017).

[33] Y. Filatov, A. V. Butenko, A. M. Kondratenko, M. A. Kondratenko, A. D. Kovalenko, and V. A. Mikhaylov, in Proceedings of the 8th International Particle Accelerator Conference (IPAC '17), Copenhagen, Denmark, 14-19 May, 2017 (JACoW, Geneva, Switzerland, 2017), paper TUPVA112, pp. 2349-2351.

[34] S. R. Amendolia, G. Batignani, G. A. Beck, E. H. Bellamy, E. Bertolucci, G. Bologna, L. Bosisio, C. Bradaschia, M. Budinich, M. Dell'orso et al., Phys. Lett. B 178, 435 (1986).

[35] G. T. Adylov, F. K. Aliev, D. Yu. Bardin, W. Gajewski, I. Ion, B. A. Kulakov, G. V. Micelmacher, B. Niczyporuk, T. S. Nigmanov, E. N. Tsyganov et al., Phys. Lett. B 51, 402 (1974).

[36] R. Reifarth and Y. A. Litvinov, Phys. Rev. ST Accel. Beams 17, 014701 (2014).

[37] T. Blum, A. Denig, I. Logashenko, E. de Rafael, B. Lee Roberts, T. Teubner, and G. Venanzoni, arXiv:1311.2198 [hep-ph].

[38] S. R. Amendolia, B. Badelek, G. Batignani, G. A. Beck, F. Bedeschi, E. H. Bellamy, E. Bertolucci, D. Bettoni, H. Bilokon, G. Bologna et al., Phys. Lett. B 146, 116 (1984).
[39] S. R. Amendolia, M. Arik, B. Badelek, G. Batignani, G. A. Beck, F. Bedeschi, E. H. Bellamy, E. Bertolucci, D. Bettoni, H. Bilokon et al. (NA7 Collaboration), Nucl. Phys. B 277, 168 (1986).

[40] J. Kahane, Phys. Rev. 135, B975 (1964).

[41] A. Akhiezer and M. Rekalo, Hadron Electrodynamics (Naukova Dumka, Kiev, 1977).

[42] W. Pauli and F. Villars, Rev. Mod. Phys. 21, 434 (1949).

[43] J. Bjorken and S. Drell, Relativistic Quantum Fields (McGrawHill, New York, 1965).

[44] A. Akhiezer and V. Berestetsky, Quantum Electrodynamics (Nauka, Moscow, 1969).

[45] D. R. Yennie, S. C. Frautschi, and H. Suura, Ann. Phys. 13, 379 (1961)

[46] E. A. Kuraev and V. S. Fadin, Sov. J. Nucl. Phys. 41, 466 (1985) [Yad. Fiz. 41, 733 (1985)].

[47] E. Tomasi-Gustafsson, G. I. Gakh, and C. Adamuscin, Phys. Rev. C 73, 045204 (2006).

[48] A. P. Kobushkin and A. I. Syamtomov, Phys. Atom. Nucl. 58, 1477 (1995) [Yad. Fiz. 58N9, 1565 (1995)].

[49] D. Abbott, A. Ahmidouch, H. Anklin, J. Arvieux, J. Ball, S. Beedoe, E. J. Beise, L. Bimbot, W. Boeglin, H. Breuer et al. (JLAB t20 Collaboration), Eur. Phys. J. A 7, 421 (2000).

[50] G. Abbiendi, C. M. Carloni Calame, U. Marconi, C. Matteuzzi, G. Montagna, O. Nicrosini, M. Passera, F. Piccinini, R. Tenchini, L. Trentadue et al., Eur. Phys. J. C 77, 139 (2017).

[51] D. Yu. Bardin, V. B. Semikoz, and N. M. Shumeiko, Yad. Fiz. 10, 1020 (1969). 